九州大学学術情報リポジトリ

Kyushu University Institutional Repository

\title{
Über Den Einfluss Der Frucht Auf Die Samenreife Bei Einigen Kulturpflanzen
}

Kosaka, Hirosi

Agronomisches Institut, Kaiserliche Kyushu-Universität

https://doi.org/10.5109/22328

出版情報: 九州大学大学院農学研究院紀要. 1 (5), pp. 197-216, 1925-10. Kyushu Imperial University バージョン：

権利関係 : 
Journal of the Department of Agriculture, Kyushu Imperial University, Vol. 1, No. 5

October 1, 1925

ÜBER DEN EINFLUSS DER FRUCHT AUF DIE SAMENREIFE BEI EINIGEN KULTURPFLANZEN'

\section{Hirosi Kosaka \\ I. EINLEITUNG}

Wir besitzen bereits eine sehr weitgehende Kenntnis der Beziehungen zwischen Samen und Früchten. Die Tatsache, dass der Samen auf die Entwickelung des Fruchtfleisches einen reizenden Einfluss hat, ist schon von Müller-Thurgau (21) bei Traubenbeeren und Kernobst gefunden worden. Nach FRUwiR'TH (8) sind die samenreichen Hülsenfrüchte schwerer als die samenarmen. GOEBEL (9) berichtete über einen beschleunigenden Einfluss der Hülsen auf das Trocknen der reifenden S.ımen. Weiterhin ist von verschiedenen Seiten über den Vorgang der Nachreife bei verschiedenen Pflanzenarten berichtet worden. Dieser Vorgang ist nicht nur bei unreif geernteten Samen $(\mathrm{I}, 2,3,4,5,6,7$, I I, I 5, 28, 3I) sondern auch bei den an abgeschnittenen Mutterpflanzen sitzenden Samen (I, 15, 19, 22, 23) mit Sicherheit festgestellt worden. Diese letztere Tatsache ist besonders bemerkenswert, weil sie, wie einige Autoren bereits betonten, $(27,32,33,34)$ für die innigen stofflichen Beziehungen zwischen Samen und Mutterpflanze von grosser Bedeutung ist. Andererseits haben zahlreiche Forschungen auch gezeigt, dass verschiedene Früchte auch nach der Trennung von ihren Mutterpflanzen in der Reifung noch weitgehende Fortschritte machen können (13, 16, 17, I8, 20, 24, 25, 26). Man misst meistens der Frucht in erster Linie eine biologische Bedeutung für den Samen zu, die in dem Samenschutz oder ihrer Aufgabe bei der Samenverbreitung zu suchen ist. Man hat aber bisher weniger Wert auf die physiologische

r. Arbeiten aus dem botanischen Laboratorium der Kaiserlichen Kiushu -Universităt, No. 8 . 
Bedeutung der Frucht für den Samen gelegt. Diesbezügliche Untersuchungen dürften aber sowohl für die Pflanzenphysiologie als auch für die Samenkunde von grosser Bedeutung sein. Ich habe deshalb seit einigen Jahren im botanischen und agronomischen Instituten mich diesen Untersuchungen gewidmet, deren Ergebnisse ich in nachfolgendem mitteilen will.

Es ist mir aufrichtiges Bedürfnis meinen Lehrern Herrn Prof. Dr. R. Kòketsu für Anregung und Leitung und den Herren Prof Dr. H. Ando und Prof. Dr. S. Kato für den mir ständig gewährten Rat und gütige Hilfe meinen herzlichsten Dank auszusprechen.

\section{MATERIAL UND METHODE}

Als Versuchsmaterial dienten die Früchte von Capsicum annuum, L., Solanum Melongena, L. und Cucumis satizus, $L$., die in unserem Versuchsgarten angebaut waren. Von Capsicum standen mir zwei Sorten : "Shishi“-und „Ômaru“, von Cucumis ebenfalls zwei Sorten: „Fushinari“" und "Nagauri" und von Solamum nur eine Sorte: „Naganasu" zur Verfügung. Im Laufe des Reifungsprozesses pflegte sich die Farbe der Capsicum-Früchte allmählich zu verändern, ich klassifizierte daher den Reifegrad der Früchte nach den Farbenabstufungen wie folgt :

I) bei Capsicum :

Reifegrad o: noch grün, aber die Früchte völlig ausgewachsen.

"I: teilweise rotbraun gefärbt.

" II : ganz rot gefärbt, das Fruchtfleisch noch hart.

" III : Fruchtfarbe wie bei II, das Fruchtfleisch aber ist hier sehr weich.

"IV: Farbe dunkelrot, es ist aber das Fruchtfleisch beinahe trocken.

Eine ähnliche progressive Veränderung der Farbe ist auch bei den Cucumis und Solanum-Früchten zu beobachten, wenn auch die Farbtöne nicht in allen Fällen übereinstimmen. Bei diesen beiden Arten war die stufenweise Klassifikation der Farbtöne sehr schwer; es gelang mir mit Sicherheit bei Cucumis und Solanum nur 3 Stufen zu unterscheiden.

2) Cucumis:

Reifegrad 0 : noch grün, aber die Früchte so ziemlich ausgewachsen. 
Reifegrad I: teilweise gelb verfärbt, die Früchte völlig ausgewachsen.

" II : gänzlich gelb vertärbt.

\section{3) Solanum:}

Reifegrad o: violett, die Früchte ziemlich gut entwickelt.

" I: teilweise gelb gefärbt, die Früchte völlig ausgewachsen.

" II : gänzlich gelb gefärbt.

Von jeder Sorte der Untersuchungsmaterialien wurden die Früchte in den genannten verschiedenen Reifegraden gleichzeitig geerntet und davon die Samen sofort oder nach verschiedener Behandlung der Früchte gesammelt, nämlich :

I) Sofort ohne jegliche Behandlung.

2) (Zim.): Teils I Woche (Zim. I), teils 2 Wochen (Zim. 2) und teils 3 Wochen (Zim. 3) lang auf einem Tische ausgebreitet.

3) (Lich.): Teils I Woche (Lich. I), teils 2 Wochen (Lich. 2) und teils 3 Wochen (Lich. 3) lang im Glashause gehalten, um die Früchte den Sonnenstrahlen auszusetzen.

4) (Wurz. Zim.): Materialien in situ auf den ausgehobenen Mutterpflanen I Woche (Wurz Zim. I), 2 Wochen (Wurz. Zim. 2) und 3 Wochen (Wurz. Zim. 3) lang auf einem Tische im Laboratorium gehalten.

5) (Wurz. Lich.): Materialien wie bei 4), aber im Glashause I Woche (Wurz. Lich. 1) 2 Wochen (Wurz. Lich. 2) und 3 Wochen (Wurz. Lich. 3) gehalten.

Als Kontrolle (Feld) liess ich auch die Früchte in allen Reifegraden auf den Mutterpflanzen auf dem Acker stehen und pflückte dieselben erst nach 2 Wochen um die Samen dann sofort ohne weitere Behandlung zu sammeln. Die Beobachtung der Farbe hatte bei allen Früchten zunächst den Zweck daraus eine Beurteilung des Reifegrades zu ermöglichen, dann wurden die Samen vom Fruchtfleisch durch Waschen getrennt. Von den ausgewaschenen Samen wurden zur Bestimmung des Trockengewichts und der Keimfähigkeit nur die sorgfältig ausgelesenen Samen benützt, während nach Grafe (IO) die Samen mit allen mit dem blossen Auge erkennbaren Abnormalitäten, und zwar diejenigen mit abnormen Formen, mit weichen Samenschalen, mit armen Inhalt oder abnormalitäten Schalenfarbe als schlechte Samen entfernt wurden. 
III. DIE BEZIEHUNGEN ZWISCHEN FRUCHT- UND SAMENFARBE

\section{a) Capsicum}

Bei Capsicum-Samen verändert sich die Farbe von "white“ zu „Lemonyellow" während des Reifungsvorganges. Es gelang mir während dieser Entwickelung 6 verschiedene Abstufungen zu unterscheiden, die ich nach der Ridgway'schen "Colorstandard and Nomenclature“ (29) bezeichnet habe, nämlich :

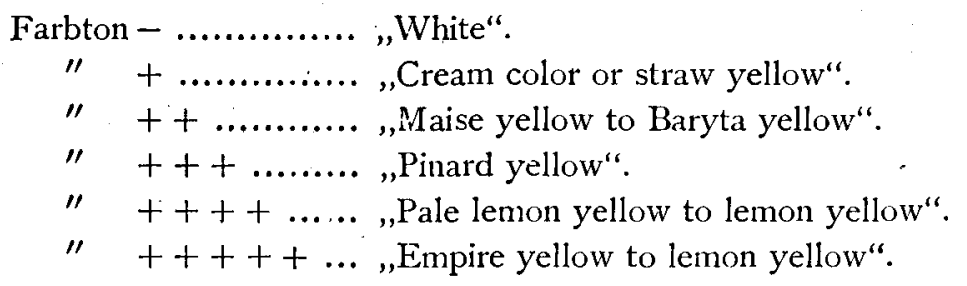

Zunächt suchte ich die Beziehungen zwischen dieser stufenweisen Veränderung der Samenfarbe und der Abtönung in der Färbung der Früchte festzustellen. Der Bequemlichkeit halber habe ich versucht den Reifegrad der Früchte zahlenmässig auszudrücken, indem ich den Reifegrad o mit I, I mit 2, II mit 3, usw. bezeichnete. Dementsprechend wurde der Farbton auch mit Zahlen ausgedrückt und zwar I für den Farbton-, 2 für,+ 3 für ++ usw.

Versuch I. Am I3. Oktober 1924. Die Capsicum-Sorte "Ômaru". Früchte in den Reifestadien o-III, Samensammlung sofort oder nach Lagerung der Früchte im Laboratorium.

Ergebnisse: in dem Falle, in dem die Samen sofort gesammelt wurden, bestand ein Parallelismus zwischen der Frucht- und der Samenfarbe, und zwar entsprachen die Farbtönen I - 4 der Samen denselben I - 4 der Früchte (Tab. I).

Tabelle I. Resultate der Beobachtungen an Capsicam-Samen, die sofort nach dem Pflücken der Frucht entnommen wurden.

\begin{tabular}{ccc}
$\begin{array}{c}\text { Reifegrade der } \\
\text { Fridchte }\end{array}$ & $\begin{array}{c}\text { Fruchtfarbe, } \\
\text { zur Zeit der Samenentnahme }\end{array}$ & $\begin{array}{c}\text { Samenfarbe, } \\
\text { o }\end{array}$ \\
I & I & 1 \\
II & 2 & 2 \\
III & 3 & 3 \\
& 4 & 4 \\
\hline
\end{tabular}

Die Resultate wurden erhalten im Mittel von Ergebnissen aus 20 Versuchsmaterialien. 
Hierzu ist noch zu bemerken, dass in den Fällen, in denen die Früchte 1-3 Wochen lang auf dem Tische im Laboratorium gelegen hatten, war deutlich zu erkennen, dass sowohl die Früchte als auch die Samen stärker gefärbt waren, obwohl die Vertiefung der Samenfarbe etwas langsamer vor sich ging, als die der Früchte ('Tab. 2).

Tabelle 2. Resultate der Beobachtungen an Capsicum-Samen bei I,agerung der Frïchte im Zimmer.

$\begin{array}{cccc}\begin{array}{c}\text { Behandlung der } \\ \text { Früchte }\end{array} & \begin{array}{c}\text { Reifegrade der Früchte } \\ \text { zur Erntezeit }\end{array} & \begin{array}{c}\text { Fruchtfarbe, } \\ \text { zur Zeit der Samenentnahme }\end{array} & \begin{array}{c}\text { Samenfarbe, } \\ \text { Zim. I }\end{array} \\ & \text { o } & 1.80 & 1.60 \\ & \text { I } & 3.00 & 2.67 \\ \text { II } & 5.00 & 3.00 \\ \text { Zim. } 3 & \text { III } & 5.00 & 3.90 \\ & 0 & 2.50 & 2.38 \\ & \text { II } & 5.00 & 3.10 \\ & \text { III } & 5.00 & 4.50\end{array}$

Die Resultate wurden erhalten im Mittel von Ergebnissen aus 20 Versuchsmaterialien.

Versuch II. Am IO. November 1924. Capsicum-Sorte "Ômaru“. Früchte in Reifestadien o, I, II. Samensammlung geschah teils sofort, teils nach Lagerung der Früchte im Zimmer oder im Glashause. Es ergab sich, dass die den Sonnenstrahlen ausgesetzten Früchte und dementsprechend auch die Samen tiefer gefärbt waren (Tab. 3).

Tabelle 3. Resultate der Beobachtungen an Capsicum-Samen der Sorte "Ômaru“.

$\begin{array}{cccc}\begin{array}{c}\text { Behandlung der } \\ \text { Frichte }\end{array} & \begin{array}{c}\text { Reifegrade der Frïchte } \\ \text { zur Erntezeit }\end{array} & \begin{array}{c}\text { Fruchtfarbe, } \\ \text { zur Zeit der Samenentnahme }\end{array} \\ \text { Sofort } & 0 & 1.00 & \text { Samenfarbe, } \\ & \text { I } & 2.00 & 3.05 \\ \text { Lich. I } & \text { II } & 3.00 & 4.00 \\ \text { Lich. 2 } & 0 & 2.73 & 1.55 \\ \text { Zim. 2 } & 0 & 3.05 & 3.32 \\ & 0 & 2.73 & 2.03 \\ \text { Iich. 3 } & \text { I } & 3.38 & 3.25 \\ & 0 & 2.63 & 4.68 \\ & \text { I } & 5.00 & 5.24 \\ & \text { II } & 5.00 & 5.24\end{array}$

Diese Resultate wurden als Mittelwerte von Ergebnissen aus 20-25 Versuchsmaterialien gefunden.

Aus diesen Untersuchungen ergibt sich, dass die Farbe der Capsicum- 
Samen in den von den Mutterpflanzen getrennten Früchten, mit dem Fortschritt der Fruchtreife tiefer wird.

Eine andere Frage ist die, ob die Farbe der Samen, die aus der Frucht genommen werden, ebenfalls eine ähnliche Änderung erfährt. Ich habe deshalb die Samen nach der Entfernung aus der Frucht einige Wochen lang im Laboratorium gehalten, um diese Frage zu beantworten. Ich führte den Versuch mit allen zu dem Versuch II gesammelten Samengruppen aus und stellte fest, dass die Samenfarbe auch ausserhalb der Früchte allmählich tiefer wird und zwar besonders deutlich in dem Falle, in dem die frisch entnommenen Samen nur wenig gefärbt sind (Tab. $4,5,6)$.

Tabelle 4. Resultate der Beobachtungen an Capsicum-Samen, die in Versuch I (Sorte "Ômaru") ausserhalb der Früchtc gehalten wurden. $\begin{array}{ccc}\text { Reifegrade der Friichte } & \text { Farbe der Samen } & \text { Farbe der Samen ausserhalb der Frucht nach } \\ \text { zur Erntezeit } & \text { zur Sammelzeit } & \text { I Woche } 2 \text { Wochen } 3 \text { Wochen }\end{array}$

\begin{tabular}{rrrrr} 
O & I.43 & 4 & 4 & 4 \\
I & 3.05 & 3 & 3 & 3 \\
II & 4.00 & 4 & 4 & 4 \\
\hline
\end{tabular}

Diese Ergebnisse wurden durch einmaligen Versuch mit 50 Samen erhalten.

Tabelle 5. Resultate der Beobachtungen an Capsicum-Samen (Sorte "Ómaru“), die zu dem in Tabelle 3 dargestellten Versuche benutzt wurden, und bei dem die Samen ausserhall der Frucht gehalten wurden.

Reifegrad der Frïchte Behandlung zur Zeit der Ernte der Friichte

\begin{tabular}{llc}
$\circ$ & Samensammmelung \\
\hline & Lich. I & 1.43 \\
$\circ$ & Lich. 2 & 1.90 \\
0 & Zim. 2 & 3.32 \\
0 & Lich. 3 & 2.05 \\
& & 4.69
\end{tabular}

Farbe der Samen Farbe der Samen, gelegt ausserhalb der Frucht

I Woche 2 Wochen 3 Wochen

\begin{tabular}{lll}
4 & 4 & 4 \\
3 & $3 \cdot 5$ & 3 \\
4 & 4.0 & 4 \\
3 & 3.0 & 3 \\
5 & 5.0 & 4 \\
\hline
\end{tabular}

Diese Ergebnisse wurden durch einmaligen Versuch mit 50 Samen erhalten.

Tabelle 6. Resultate derselben Bcobachtungen wie in Tabelle 5, jedoch mit dem Reifegrad I. Reifegrad der Früchtc
zur Lrntezeit

I

I

I
Behandelung
der Früchte

Sofort

Zim. 2

Lich. 3
Farbe der Samen zur Sammelzeit

3.05

3.25

5.24
Farbe der Samen, gelegt ausserhalb der Frucht $A$ the I Woche : 2 Wochen 3 Wochen

$\begin{array}{lll}3 & 3 & 30 \\ 4 & 4 & 4.0 \\ 6 & 5 & 4.5\end{array}$


Versuch III. Den Versuchen I und II gleichsinnige Versuche wurden auch mit Capsicum-Früchten der Sorte „Shishi“ ausgeführt, deren Früchte am 20. November 1924, geerntet wurden. Die Ergebnisse, die in Tabelle 7 zusammengefasst sind, sind den vorhergehenden ähnlich.

Tabelle 7. Resultate der Vcrsuche mit Catsicum-Sorte „Shishi“.

\begin{tabular}{|c|c|c|c|c|}
\hline $\begin{array}{l}\text { Behandelung } \\
\text { der Fridchte }\end{array}$ & $\begin{array}{l}\text { Reifegrade der } \\
\text { Früchte zur } \\
\text { Zeit der Ernte }\end{array}$ & $\begin{array}{r}\text { Fruchttarb } \\
\text { zur Zeit }\end{array}$ & $\begin{array}{l}\text { amenfarbe } \\
\text { Samen- } \\
\text { lung }\end{array}$ & $\begin{array}{l}\text { Farbe der Samen nach } 2 \text { Wochen } \\
\text { langer Lagerung ausscrhalb } \\
\text { der Frucht }\end{array}$ \\
\hline \multirow[t]{3}{*}{ Sofort } & -0 & $\mathrm{x} . c 0$ & 1.56 & 4 \\
\hline & $\mathbf{I}$ & 2.00 & 3.00 & 4 \\
\hline & II & 3.00 & 3.80 & 4 \\
\hline \multirow[t]{2}{*}{ Zim. 2} & o & 2.05 & 1.63 & 4 \\
\hline & I & 3.67 & 3.50 & 4 \\
\hline \multirow[t]{2}{*}{ I,ich. 2} & o & 3.26 & 2.16 & 4 \\
\hline & I & 4.00 & 3.88 & 4 \\
\hline 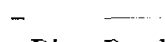 & $-\cdots$ & $\cdots \cdot-\cdots$ & $\cdots \quad-\cdots \cdot-$ & $\ldots ;-\cdots--\cdots-$ \\
\hline
\end{tabular}

Diese Resultate wurden durch Beobachtungen an je 30 Friichten und je 50 Samen erhaten.

Aus allen vorhergehenden Versuchen geht hervor, dass die fortschreitende Verfärbung der Capsicum-Samen stets eintritt, gleichgiltig ob in oder ausserhalb der Frucht. Nun bliebe aber anzunehmen, dass derselbe Vorgang da weit deutlicher vor sich geht, wo die Früchte in situ auf den mit den Wurzeln ausgehobenen Mutterpflanzen oder auf den im Acker im natürlichen Zustande sich entwickelnden Pflanzen sich befinden. Ich habe deshalb diesbezügliche Versuche angestellt.

Versuch IV. Gleichzeitig mit dem Versuch III wurden von demselben Material der Sorte „Shishi“ einige Mutterpflanzen mit den Wurzeln ausgehoben und die Früchte daran belassen. Diese wurden entweder im Laboratorium oder im Glashause aufbewahrt. Als Kontrolle liess ich andere Pflanzen im Ackerboden stehen und pfückte die Früchte erst zwei Wochen später, um die Samen als Kontrolle zu benützen. Um den Fortschritt in der Samenreife zu beobachten, wurden auch hier die Samen nach der Entfernung aus der Frucht noch zwei Wochen lärger im Laboratorium gehalten. Durch diese Versuchsreihe wurde festgestellt, dass die Färbung der Samen innerhalb der an den Mutterpflanzen sitzenden Früchte in fast demselben Grade fortschreitet wie w. o. dargestellt, wenn auch die Geschwindigkeit des Farbenwechsels der Früchte selbst nicht immer übereinstimmte. Bei den natürlich im Ackerboden sich entwickelnden Individuen ging diese Frscheinung bedeutend langsamer vor sich (Tab. 8). 
Tabelle 8. Resultate der Versuche erhalten mit einer Sorte von Capsicum "Shishi“.

\begin{tabular}{ccccc}
$\begin{array}{c}\text { Behandelung } \\
\text { der Früchte }\end{array}$ & $\begin{array}{c}\text { Reifegrade der } \\
\text { Früchte zur } \\
\text { Erntezeit }\end{array}$ & \multicolumn{2}{c}{$\begin{array}{c}\text { Fruchtfarben, Samenfarben, } \\
\text { bei der Samensammelung }\end{array}$} & $\begin{array}{c}\text { Farbe der Samen nach } 2 \text { Wochen } \\
\text { langer Lagerung ausserhalb } \\
\text { der Frucht }\end{array}$ \\
Feld. 2 (Kontrolle) 0 & 1.45 & 2.35 & 4 \\
& I & 2.50 & 3.00 & 4 \\
Wurz. Zim. 2 & 0 & 2.33 & 2.12 & 4 \\
Wurz. Lich. 2 & 0 & 2.83 & 2.58 & 4 \\
& 1 & 4.00 & 3.83 & 4
\end{tabular}

Resultate der Beobachtungen, die an 25-30 Friichten und je 5o Samen gemacht wurden.

\section{b. Cucumis}

Ein ähnlicher Versuch wurde auch mit Früchten einer Sorte von Cucumis „Nagauri“, ausgefühtt, welche am 6 . Juni I924, geerntet wurden, und es wurde bestätigt, dass die Farbe der Samen sich von „Reed yellow" zu „Cream color“" während ihres Reifungsvorgangs verändert hatte. In näherem konnte ich die Farbtöne nach der RIDGWAY'schen Nomenklatur (29) in folgenden 3 Graden unterscheiden.

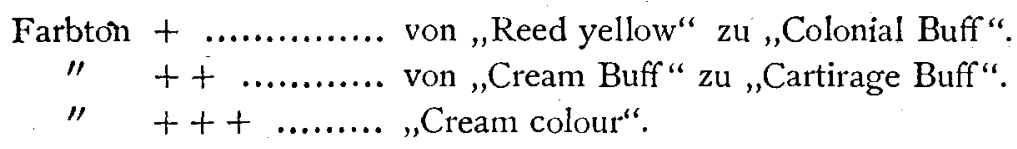

Auch hierbei wurden sowohl die Farbe der Frucht als auch die der Samen der Bequemlichkeit halber zahlenmässig angegeben, und zwar bei der fruchtfarbe I für den Reifegrad 0,2 für den I, usw.; und bei der Samenfarbe I für den Farbton,+ 2 für den,++ 3 für den +++ . Aus den Versuchen war zu ersehen, dass eine fortschreitende Farbänderung der Samen mit der Änderung der Fruchtfarben Hand in Hand ging. Die Ergebnisse sind in der Tabelle 9 zusammengesetzt.

Tabelle 9. Resultate der Versuche erhalten mit einer Sorte von Cucumis „Nagauri“.

$\begin{array}{cccc}\begin{array}{c}\text { Behandelung } \\ \text { der Frïchte } \\ \text { Sofort }\end{array} & \begin{array}{c}\text { Reifegrade der Frichte } \\ \text { zur Erntezeit }\end{array} & \begin{array}{c}\text { Fruchtfarbe, Samenfarbe, } \\ \text { bei der Samensammelung }\end{array} \\ & \text { o } & \text { I } & \text { I } \\ \text { Zim. I } & \text { I } & 2 & 2 \\ & \text { II } & 3 & 3 \\ \text { Zim. 2 } & \text { o } & 3 & \text { I } \\ & \text { I } & 2 & 3 \\ & \text { II } & 3 & 3 \\ & \text { o } & 3 & 3 \\ \text { I } & 3 & 3 \\ & \text { II } & 3 & 3\end{array}$




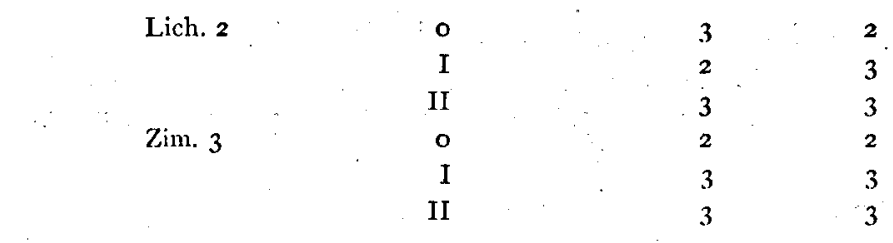

Resultate von Beobachtungen, die an 7-10 Friichten und je 5o Samen gemacht wurden.

\section{Solanum}

Bei Solanum-Samen waren nur zwei Farbtöne zu unterschiedbar, von welchen die eine den unreifen und die andere den reifen Samen entspricht. Daher war es unmöglich von der Farbtönen auf den Reifegrade der Samen zu schliessen. Andererseits war auch die Farbänderung der von der Mutterpflanze getrennten Früchte nur geringfügig, obwohl man auch hierbei das Vorhandensein einer ähnlichen Neigung der Farbänderung wie bei Capsicum oder Cucumis finden kann. Eingehende diesbezügliche Untersuchungen mit diesem Material wurden also aufgelassen.

\section{EINFLUSS DER FRUCHT AUF DIE TROCKENGEWICHTSZUNAHME DER SAMEN}

Durch die vorhergehenden ist festgestellt, dass die Früchte wenigstens in gewissem Umfange einen Einfluss auf die Verfärbung der darin liegenden Samen ausübt. $\mathrm{Ob}$ die Färbung der Samen auf die Reifung derselben hinweist, kann aber nicht ohne weiteres behauptet werden. Ich habe deshalb die Veränderungen in dem Trockengewicht der Samen verfolgt, um Klarheit in diese Frage zu bingen. In allen vorher beschriebenen Versuchen wurde auch das Trockengewicht der Samen bestimmt. Für Trocknung des Materials wurde hierbei ein elektrothermischer Trockenschrank bei einer Temperatur von $100-102^{\circ} \mathrm{C}$. gebraucht, und das Material wurde 24 Stunden lang im Schrank gehalten. Bei der „Nagauri“-, und. der ,Fushinari"-Sorte von Cucumis wurden immer Ioo Samen-Körner, bei der "Ômaru"- und der "Shishi"-Sorte von Capsicum stets 200 Samen-Körner, und bei der „Naganasu“-Sorte von Solanum 500 SamenKörner gebraucht.

\section{a. Capsicum}

Versuch V: Versuch mit den Samen der Capsicum-Sorten „Ômaru“ und „Shishi“. Resultate : Das Trockengewicht der Samen innerhalb der Früchte nimmt stets zu, ganz gleich ob die Samen sich in den Früchten 
auf den im Acker sich bewurzelten oder mit den Wurzeln ausgehobenen Mutterpflanzen oder in den geplückten befinden. Selbstverständlich ist diese Erscheinung nur bei den jüngeren Samen ersichtlich, weil die Gewichtszunahme der Samen, bei clenen, der Reifungsprozess schon weiter fortgeschritten ist, nicht weiter gehen kann (Tab. IO, II, 12).

Tabelle 10." Trockengewichts-Bestimmung von Samen der Copsicum-Sorte "Ômaru", die zu dem Versuch I benutzt wurden.

\begin{tabular}{|c|c|c|c|c|c|c|c|c|}
\hline \multirow[b]{2}{*}{$\begin{array}{l}\text { Behandelung } \\
\text { der Früchte }\end{array}$} & \multicolumn{5}{|c|}{ Absolutes Trockengewicht } & \multicolumn{3}{|c|}{ in Ratio } \\
\hline & $\begin{array}{l}\text { Reifegrade der } \\
\text { Friichte zur } \\
\text { Erntezeit }\end{array}$ & $\begin{array}{c}\text { o } \\
\text { gr. }\end{array}$ & $\begin{array}{c}\text { I } \\
\text { gr. }\end{array}$ & $\begin{array}{l}\text { II } \\
\text { gr. }\end{array}$ & $\begin{array}{l}\text { III } \\
\text { gr. }\end{array}$ & o & $\boldsymbol{I}$ & II \\
\hline Sofort & & 0.7929 & I. I $3 \mathrm{CO}$ & 1.0862 & I. 1184 & 100 & 143 & I 37 \\
\hline Zin. I & & 0.6716 & 1.1303 & $1.155^{\circ}$ & 1.0940 & 85 & 143 & 146 \\
\hline Zim. 3 & & 0.8496 & 一 & 1.1423 & 1.0992 & 107 & - & 144 \\
\hline
\end{tabular}

- Tabelle I I. Trockengewichts-Bestimmung von Samen der Capsicum-Sorte „Ömaru“, die zu dem Versuch II benutzt wurden.

$$
\text { Absolutes Trockengewicht in Ratio }
$$

$\begin{array}{cccccccc}\begin{array}{c}\text { Behandelung } \\ \text { der Früchte }\end{array} & \begin{array}{c}\text { Reifegrade der } \\ \text { Früchte zur } \\ \text { Erntezeit }\end{array} & \begin{array}{c}0 \\ \text { gr. }\end{array} & \begin{array}{c}\text { I } \\ \text { gr. }\end{array} & \begin{array}{c}\text { II } \\ \text { gr. }\end{array} & 0 & \text { I } & \text { II } \\ \text { Sofort } & & 0.5637 & 0.9970 & 1.1798 & 100 & 177 & 209 \\ \text { Lich. 1 } & & 0.7242 & - & - & 129 & - & - \\ \text { Lich. 2 } & & 0.8132 & - & - & 144 & - & - \\ \text { Zim. 2 } & & 0.8188 & 1.0252 & - & 145 & 182 & - \\ \text { Lich. 3 } & & 0.8346 & 0.9935 & 1.1739 & 148 & 176 & 208\end{array}$

Tabelle 12. Trockengewichts-Bestimmung von Samen der Capsicum-Sorte „Shishi“, die zum Versuch III benutzt wurden.

\begin{tabular}{|c|c|c|c|c|}
\hline & \multicolumn{2}{|c|}{ Absolutes Trockengewicht } & \multicolumn{2}{|c|}{ in Ratio } \\
\hline $\begin{array}{c}\text { Behandelung } \\
\text { der Frïchte }\end{array} \backslash \begin{array}{c}\text { Reifegrade.der } \\
\text { Früchte zur } \\
\text { Erntezeit }\end{array}$ & $\begin{array}{c}\mathbf{0} \\
\text { gr. }\end{array}$ & $\underset{\text { gr. }}{\text { I }}$ & 0 & I \\
\hline Sofort & 0.8029 & 1.4031 & 100 & 175 \\
\hline Feld. 2 (Kontrolle) & I.0455 & $1.3^{642}$ & 130 & 170 \\
\hline Zim. 2 & 0.8704 & I. 34,55 & 108 & 168 \\
\hline Lich, 2 & 0.9717 & 1.2036 & 121 & 150 \\
\hline Wurz. Zim. 2 & 1.0077 & - & 126 & - \\
\hline Wurz. Lich. 2 & 1.0280 & 1.4060 & 128 & 175 \\
\hline
\end{tabular}

b. Cucumis

Versuch VI. Ein dem Versuche V gleichsinniger Versuch, mit dem - Cucumis-Sorten "Nagauri“ und „Fushinari“. Resultate: dieselben wie beim Versuch V, aber weit deutlicher (Tab. I 3 und 14). 
Tabelle 13. Trockengewichts-Bestimmung von Samen der Cucumis-Sorte „Nagauri“, deren Früchte am 6. Juli 1924, geerntet worden waren.

\begin{tabular}{|c|c|c|c|c|c|c|}
\hline \multirow[b]{2}{*}{$\begin{array}{c}\text { Behandelung } \\
\text { der Früchte }\end{array} \quad \begin{array}{c}\text { Reifegrade der } \\
\text { Frifchte zur } \\
\text { Erntezeit }\end{array}$} & \multicolumn{3}{|c|}{ Absolutes Trockengewiclit } & \multicolumn{3}{|c|}{ in Ratio } \\
\hline & $\begin{array}{c}\text { o } \\
\text { gr. }\end{array}$ & $\begin{array}{l}\text { I } \\
\text { gr. }\end{array}$ & $\begin{array}{l}\text { II } \\
\text { gr. }\end{array}$ & 0 & I & II \\
\hline Sofort & 1.07 .38 & 2.9116 & 3.0301 & 100 & 271 & 282 \\
\hline Zim. I & I.9149 & 2.5708 & 2.7983 & 178 & 239 & $26 r$ \\
\hline Zim. 2 & 1.9401 & $2.346 \mathrm{I}$ & 3.0311 & 181 & 218 & 282 \\
\hline Zim. 3 & 2.1559 & 2.3493 & 2.9609 & 201 & 219 & 276 \\
\hline
\end{tabular}

Tabelle 14. Trockengewichts-Bestimmung von Samen der Cucumis-Sorte „Fushinari“, deren Früchte am I8. Juli 1924, geerntet worden waren.

\begin{tabular}{|c|c|c|c|c|c|c|c|}
\hline \multirow[b]{2}{*}{$\begin{array}{c}\text { Behandelung } \\
\text { der Früchte }\end{array}$} & & \multicolumn{3}{|c|}{ Absolutes Trockengewicht } & \multicolumn{3}{|c|}{ in Ratio } \\
\hline & $\begin{array}{l}\text { Reifegrade der } \\
\text { Früchte zur } \\
\text { Erntezeit }\end{array}$ & $\begin{array}{c}0 \\
\text { gr. }\end{array}$ & $\begin{array}{c}\text { I } \\
\text { gr. }\end{array}$ & $\begin{array}{l}\text { II } \\
\text { gr. }\end{array}$ & 0 & I & II \\
\hline Sofort & & 0.5710 & 0.5774 & 0.9144 & 100 & IOI & 335 \\
\hline Lich. I & & - & 0.8237 & $\mathrm{I} .5485$ & - & 144 & $27 \mathrm{I}$ \\
\hline Lich. 2 & & - & I. 3805 & 1.6964 & - & 242 & 297 \\
\hline Lich. 3 & & - & - & 1.3709 & - & - & 240 \\
\hline Zim. 3 & & 2.5462 & 2.2920 & 2.1570 & 446 & 401 & 378 \\
\hline
\end{tabular}

\section{c. Solanum}

Versuch VII. Versuch mit der Solanum-Sorte „Naganasu“. Resultate : Ähnlich wie bei Cucumis (Tab. I5).

Tabelle 15. Untersuchungsresultate erhalten mit der Solmnim-Sorte „Naganasu“, deren Früchte am 8. Oktober 1924, geerntet wurden.

\begin{tabular}{|c|c|c|c|c|c|c|c|}
\hline . & & \multicolumn{3}{|c|}{ Absolutes Trockengewicht } & \multicolumn{3}{|c|}{ in Ratio } \\
\hline $\begin{array}{c}\text { Behandelung } \\
\text { der Früchte }\end{array}$ & $\begin{array}{l}\text { Reifegrade der } \\
\text { Friichte zur } \\
\text { Erntezeit }\end{array}$ & $\begin{array}{c}0 \\
\text { gr. }\end{array}$ & $\underset{\text { gr. }}{\text { I }}$ & $\begin{array}{l}\text { II } \\
\text { gr. }\end{array}$ & 0 & $\mathbf{I}$ & II \\
\hline Sofort & & 1.0493 & 1.4545 & 2.2065 & 100 & I 39 & 210 \\
\hline Zim. I & & 1.4568 & I. 8803 & - . & 139 & 179 & - \\
\hline Lich. 2 & & 1.2732 & 1.7828 & 2.6220 & $\mathbf{I} 2 \mathbf{1}$ & 170 & 250 \\
\hline
\end{tabular}

Aus allem Gesagten geht also hervor, dass das Trockenwegicht der unreifen Samen in dem Falle zunimmt, wo sie in den Früchten der mit Wurzel aufgehobenen Mutterpflanzen liegen und selbst da, wo sie innerhalb der abgeplückten Früchte liegen bleiben. Aller Wahrscheinlichkeit nach wandern also die Baustoffe während der Fruchtlagerung aus der Frucht her in die Samen. Als WAssilief (32) einen analytischen Versuch über 
die Eiweisbildung in reifenden Legminosen-Samen anstellte, hielt er es sehr wahrscheinlich, dass die Einwanderung von Eiweisstickstoff aus den Hülsen in die Samen stattfindet. Andererseits gibt es in der Literatur (13, 16, 17, 18, 20, 24, 25, 26, etc.) vielfache Angaben darüber, dass bei Lagerung verschiedener Obstfrüchte die reduzierenden Zuckerarten beträchtlich zunehmen. Nach JANSON (I4) soll der Kohlenhydratgehalt in Hafer- und Gcrste-Samen sehr arm sein, wenn die Mutterpflanzen früzeitig im Reifungsprozess abgeschnitten werden. Obwohl es uns in unserm Falle nicht ohne weiteres klar ist, welche Stoffe von der Frucht in die Samen treten, so ist doch anzunehmen, dass Baustoffe wie die Kohlenhydrate oder Eiweisstoffe hier eine besondere Rolle spielen.

Die Untersuchungen, welche Siegrit (30) an Soinmer-Weizen, Lacanus (19) an Roggen, Nowacki (23) an Weizen, Ando (1) an Reis und JANSON (I4) an Hafer und Gerste ausführten, erbrachten ein scheinbar ähnliches Resultat; und zwar konstatierten sie, dass das Gewicht der Samenkörner durch die Lagerung ohne Trennung von den abgeschnittenen Mutterpflanzen deutlich zunahm. Doch aus allem diesem können wir nicht sicher auf einen Einfluss der Frucht auf die aus den Früchten in die Samen gerichtete Stoffeinwanderung schliessen, weil die genannten Versuchsmaterialien anderer Autoren in allen Fällen fleischarme GramineenFrüchte waren. Die Sache liegt also ganz anders als in unseren Fällen. Merkwürdig ist hier ferner, dass der stoffliche Einfluss der Frucht auf die Samen bei den fleischreichen Früchten von Cucumis und Solanum deutlicher war, als bei den fleischarmen von Capsicum.

\section{EINFLUSS DER FRUCHT AUF DIE KEIMFÄHIGKEIT DER SAMEN}

Durch die erwähnten Versuche ist erwiesen, dass während der Fruchtlagerung, seien sie auf dem Mutterpflanzen befindlich oder davon getrennt, eine Stoffeinwanderung von Frucht in die Samen stattfindet. Eine andere Frage ist, ob diese Stoffeinwanderungserscheinung den Reifungsprozess der Samen begleitet. Um das zu erklären, habe ich weiter einige Versuche über die Keimfähigkeit der Samen ausgeführt. Alle Versuchsgruppen von Samen, die teilweise zur Bestimmung der Gewichtszunahme gebraucht wurden, wurden in ein Keimbett gebracht und ihre Keimfahigkeit bestimmt. Die Keimversuche mit Capsicum wurden immer im Thermostaten bei $20^{\circ} \mathrm{C}$. und mit Cucumis immer bei Zimmertemperatur $\left(20-25^{\circ} \mathrm{C}\right.$.) gemacht; die Versuche erstreckten sich über eine Zeit von 1o Tagen Als Keimbett wurde in der Mehrzahl der Fälle der LiebenBERG'sche Apparat gebraucht. Weil aber die Solanum-Samen in diesem 
Keimbette schwer keimfähig waren, habe ich dafür eine grosse Schale benutzt, in der Quarzsand ständig mit demselben Volumen Wasser gesättigt war. Die Apparate mit den ein gesäten Samen wurden zuerst auf 7 Tage bei $30^{\circ} \mathrm{C}$. im Thermostat gehalten, dann in's Glass-Haus gebracht, wo sich die Beobachtung über 10 Tage erstreckte.

\section{a. Capsicum}

Versush VIII. Keimungsyersuch mit Samen der Capsicum-Sorten „Shishi“" und „Ômaru“. Die Resultate: die Keimfähigkeit der Samen ausgedruckt in $\%$ nahm durch die Lagerung der Frucht zu, einerlei, ob die Frucht auf der Mutterpflanze belassen worden oder davon getrennt worden war; und zwar war das Keimfahigkeit der Samen desto grösser, je tiefer die Früchte gefärbt waren. In näheren war die Keimfähigkeit der aus den besonnten Früchten gesammelten Samen weit höher als die der Samen aus den im Zimmer gelagerten Früchten (Tab. I6, i 7 und i 8).

Tabelle I6. Resultate der Bestimmung der Keimfähigkeit von Caficum-Samen (Sorte "Ômaru“), welche teilwcise im versuch V gebraucht wurden.

\begin{tabular}{|c|c|c|c|c|c|}
\hline \multirow[b]{2}{*}{$\begin{array}{l}\text { Behandelung } \\
\text { der Frïchte }\end{array}$} & \multirow[b]{2}{*}{$\begin{array}{l}\text { Reifegrade der } \\
\text { Früchte zur } \\
\text { Erntezeit }\end{array}$} & \multicolumn{4}{|c|}{ Keimprozente } \\
\hline & & 0 & 1 & II & III \\
\hline Sofort & & 3.0 & $5 \cdot 5$ & 645 & 96.5 \\
\hline Zim. I & & 9.5 & 84.0 & 95.5 & 97.5 \\
\hline Zim. 3 & & 87.0 & - & 96.5 & 97.5 \\
\hline
\end{tabular}

Die Daten sind Mittelwerte von zweimaligen Versuchen mit je Ioo Samen.

Tabelle 17. Resultate erhalten mit Capsicum Samen (Sorte "Ômaru"), welche teilweise im Versuch V. gebraucht wurden.

$\begin{array}{ccccc}\begin{array}{c}\text { 13ehandelung } \\ \text { der Frichte }\end{array} & \begin{array}{c}\text { Reifegrade der } \\ \text { Früchte zur } \\ \text { Erntezeit }\end{array} & 0 & \text { Keimprozente } & \text { II } \\ \text { Sofort } & 1.00 & 15.00 & 55.67 \\ \text { Lich. I } & 9.67 & - & - \\ \text { Lich. 2 } & 75.00 & - & - \\ \text { Zim. 2 } & \mathbf{2 2 . 3 3} & 67.67 & - \\ \text { Lich. 3 } & 63.67 & 88.67 & 96.67\end{array}$

Die Daten sind Mittelwerte von dreimaligen Versuchen, ausgeführt mit je 100 Samen. 
Tabelle 18. Resulte erhalten mit Capsicum-Samen (Sorte „Shishi“), welche im Versuch $\mathrm{V}$ benutzt wurden.

\begin{tabular}{|c|c|c|}
\hline & \multicolumn{2}{|c|}{ Keimprozente } \\
\hline $\begin{array}{c}\text { Behandelung } \\
\text { der Frïchte }\end{array} \quad \begin{array}{c}\text { Reifegrade der } \\
\text { Friichte zur } \\
\text { Erntezeit }\end{array}$ & 0 & I \\
\hline Sofort & 0.67 & 33.50 \\
\hline Feld. 2 (Kontrolle) & 16.33 & 14.33 \\
\hline Zim. 2 & 53.67 & 60.33 \\
\hline Lich. 2 & 62.33 & 97.00 \\
\hline Wurz̀ Zim. 2 & - 62.67 & - \\
\hline Wurz Lich. 2 & 68.67 & 96.67 \\
\hline
\end{tabular}

Die Daten sind Mittelwerte von dreimaligen Versuchen, ausgeführt mit je Ico Samen.

Versuch IX. Versuch mit Samen, die zwecks Prüfung der sogenannten Nachreifung der Samen nach der Ernte noch weiter ausserhalb der Frucht einige Wochen lang im Zimmer gelegen hatten. Es ergab sich dass, obwohl eine Keimfähigkeit in gewissem Umfange auch der Samen ausserhalb der Frucht bemerkbar war, diese doch sehr viel geringer war als bei den Samen, die innerhalb der Frucht gelagert worden waren (Tab. 19, 20, 21 und 22).

Tabelle 19. Versuchsresultate erhalten mit Samen aus den Früchten mit dem Reifegrad o von Capsicum (Sorte "Ômaru"), welche teilweise in den Versuch VIII gebraucltt wurden.

\begin{tabular}{cccccc}
$\begin{array}{c}\text { Reifegrad der } \\
\text { Frichte zur } \\
\text { Erntezcit }\end{array}$ & $\begin{array}{c}\text { Behandelung } \\
\text { der Früchte }\end{array}$ & \multicolumn{4}{c}{ Keimprozente der Samen, } \\
sammelungszeit & gelagert ausserhall der Frucht \\
I Woche & 2 Wochen & 3 Wochen \\
0 & Sofort & 3.0 & II & I5 & 2I \\
0 & Zim. I & 9.5 & 8 & 7 & 10 \\
o & Zim. 3 & 87.0 & 95 & 87 & 92 \\
\hline
\end{tabular}

Die Daten sind aus je einem Versuch ausgefiihrt mit je soo Samen.

Tabelle 20. Versuchsresultate erhalten an den aus den Früchten mit dem Reifegrad II gesammelten Samen vón Capsicum (Sorte „Ômaru"), welche teilweise in dem Versuch VIII benutzt wurden.

\begin{tabular}{cccccc}
$\begin{array}{c}\text { Keifcgrad der } \\
\text { Friichte zur } \\
\text { Erntczeit }\end{array}$ & $\begin{array}{c}\text { Behandelung } \\
\text { der Früchte }\end{array}$ & $\begin{array}{c}\text { Kur Samen- } \\
\text { sammelungszeit }\end{array}$ & $\begin{array}{c}\text { gelagert ausserhalb der Frucht } \\
\text { 1 Woche }\end{array}$ & 2 Wochen & 3 Wochen \\
II & Sofort & 64.5 & 70 & 82 & 8 I \\
II & Zim. I & 95.5 & 99 & 97 & 98 \\
II & Zim. 3 & 96.5 & 96 & 100 & 98 \\
\hline
\end{tabular}

Die Daten sind die Resultate von je einem Versuche ausgeführt mit je roo Samen. 
Tabelle 2I. Resultate gefunden an den aus den Früchten mit dem Reifegrad o gesammelten Samen von Capscum (Sorte "Ômaru"), welche teilweise in dem Versuch VIII gebraucht wurden.

\begin{tabular}{|c|c|c|c|c|c|}
\hline \multirow{2}{*}{$\begin{array}{l}\text { Reifegrad der } \\
\text { Früchte zur } \\
\text { Erntezeit }\end{array}$} & \multirow{2}{*}{$\begin{array}{l}\text { Behandelung } \\
\text { der Frichte }\end{array}$} & \multicolumn{3}{|c|}{ Keimprozente der Samen, } & \multirow[b]{2}{*}{$\begin{array}{l}\text { der Frucht } \\
3 \text { Wochen }\end{array}$} \\
\hline & & $\begin{array}{c}\text { zur Samen- } \\
\text { sammelungszeit }\end{array}$ & $\begin{array}{l}\text { gelagert } \\
\text { I Woche }\end{array}$ & $\begin{array}{l}\text { ausserhalb } \\
2 \text { Wochen }\end{array}$ & \\
\hline 0 & Sofort & 1.00 & 0 & 2 & 0 \\
\hline 0 & Iich. I & 9.60 & 15 & 12 & 17 \\
\hline o & I,ich. 2 & 75.00 & 65 & 67 & 67 \\
\hline 0 & Zim. 2 & 22.33 & 20 & 17 & 28 \\
\hline o & Lich. 3 & 63.67 & 71 & 71 & 72 \\
\hline
\end{tabular}

Die Daten sind die Resultate von je einem Versuche ausgeführt mit je rco Samen.

Tabelle 22. Resultate erhalten an den aus den Früchten mit dem Reifegrad o gesammelten Samen von Capsicum (Sorte „Shishi"), welche teilweise in dem Versuch VIII benutzt wurden.

Reifegrad
Friichte
Erntezei
0
0
0
0
0
0

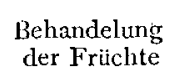

Sofort

Feld. 2

Zim. 2

Lich. 2

Wurz. Zim. 2

Wurz. Lich. 2
Keimprozente der Samen,

zur Samen-

0.67

gelagert, ausserhalb der Frucht 2 Wochen lang

\section{o}

9.33

46.33

65.50

42.25

67.00

Die Daten sind die Mittelwerte von dreimaligen Versuchen ausgefïhrt mit je roo Samenkörnem.

\section{b. Cucumis}

Die gleichen Keimungsversuche wie bei Capsicum wurden' danach mit Cucumis-Samen wiederholt. Dass die Keimfähigkeit der in den gelagerten Früchten befindlichen Samen zunimmt, war auch bei diesem Material deutlich zu sehen (Tab. 23 und 24).

Tabelle 23. Bestimmung der Keimfähigkeit bei den Samen von Cucumis (Sorte „Nagauri"), welche teilweise im Versuch VI benutzt worden waren.

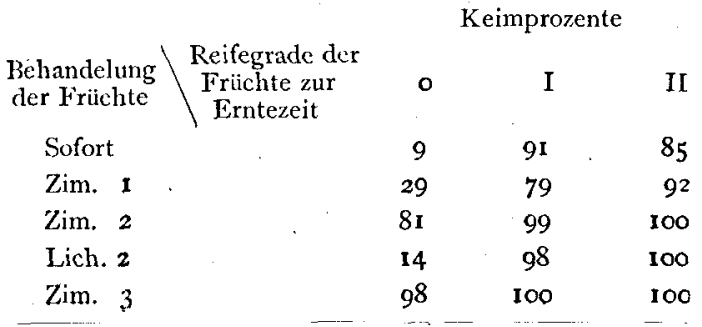

Die Daten sind die Mittelwerte von dreimaligen Versuchen ausgefihrt mit je 5o Samenkörnern. 
Tabelle 24. Bestimmung der Keimfähigkeit bei den Samen von Cuczmmis (Sorte „Fushinari“"), welche teilweise im Versuch VI gebraucht wurden.

$\begin{array}{cccc}\begin{array}{c}\text { Behandelung } \\ \text { der Früchte }\end{array} & \begin{array}{c}\text { Reifegrade der } \\ \text { Frïchte zur } \\ \text { Erntezeit }\end{array} & \text { I } & \text { II } \\ \text { Sofort } & & 0 & 75 \\ \text { Lich. I } & & 6 & 83 \\ \text { Lich. 2 } & & 70 & 88 \\ \text { Lich. 3 } & . & 88 & 78 \\ \text { Zim. 3 } & & 99 & 98\end{array}$

Die Daten sind die Mittelwerte von zweimalıgen Versuchen ausgeführt mit je 50 Samen.

Weil aber bei dem Cucumis-Falle eine fast gleichgradige Zunahme der Keimprozente auch bei den ausserhalb der Früchte gelegten Samen konstatierbar war, so scheint es uns, dass die an den innerhalb der Früchte befindlichen festgestellte Tatsache nichts anders als der sog. Nachreifungsprozess der Samen ist (Siehe Tab. 25). Erinnern wir uñs aber der Tatsache, dass bei den innerhalb der Frucht befindlichen Samen eine deutliche Zunahme des Trockengewichtes konstatierbar war (Siehe Tab. I 3), so können wir schliessen, dass auch bei diesem Material die Samen innerhalb der Frucht besser reiften, als die ausserhalb der Frucht.

Es sei hinzugefügt, dass bei diesem Material die Zunahme der Keimfähigkeit der aus den besonnten Früchten gesammelten Samen weit niedriger war, als die der aus den im Zimmer gelagerten Früchten genommenen Samẹn (Siehe Tab. 24 und 25).

Tabelle 25. Resultate erhalten an den aus den Früchten mit dem Keifegrad o gesam. melten Samen von Cucumis (Sorte „Nagauri"), welche teilweise in Tahelle 23 gebraucht worden waren.

\begin{tabular}{|c|c|c|c|c|c|c|}
\hline $\begin{array}{l}\text { Reifegrad der } \\
\text { Frïchte zur } \\
\text { Erntezeit }\end{array}$ & $\begin{array}{l}\text { Behandelung } \\
\text { der Frïchte }\end{array}$ & \multicolumn{5}{|c|}{$\begin{array}{l}\text { Keimprolente der Samen, } \\
\text { gelagert ausserhalb der Frucht, } \\
\text { it I Woche } 2 \text { Wochen } 3 \text { Wochen } 8 \text { Wochen }\end{array}$} \\
\hline 0 & Sofort & 9 & 50 & 34 & 22 & 43 \\
\hline 0 & Zim. I & 29 & 76 & 82 & - & - \\
\hline 0 & Zim. 2 & $8 I$ & 90 & - & - & 71 \\
\hline 0 & Zim. 3 & 98 & - & - & - & 96 \\
\hline
\end{tabular}

Die Daten sind die Resultate von 2 maligen Versuchen ausgeführt mit je 40-50 Samenkörnern.

\section{c. Solanum}

Gleichsinnige Versuche wurden weiter mit Solanum-Samen ausgeführt. Die positiven Resultate traten hierbei ähnlich oder vielmehr deutlicher in 
Erscheinung als bei Capsicum, d.h. es war die Zunahme der Keimfähigkeit der in der Frucht enthaltenen. Samen merkwürdig; während die Zunahme der Keimfahigkeit bei den ausserhalb der Frucht gelegten Samen kaum bemerkbar war (Tab. 26, 27, 28). Die Keimfähigkeit der aus den den Sonnenstrahlen ausgesetzten Früchten genommenen Samen war. hierbei beinahe gleich derselben der aus den im Zimmer gelagerten Früchten gesammelten Samen.

Tabelle 26. Untersuchungsrésultate erhalten mit Samen von Solamum (Sorte „Naganasu"), deren Früchte am 10. September 1924 geerntet wurden.

$\begin{array}{lcccc}\begin{array}{c}\text { Behandelung } \\ \text { der Früchte }\end{array} & \begin{array}{c}\text { Reifegrade der } \\ \text { Früchte zur } \\ \text { Erntezeit }\end{array} & 0 & \text { I } & \text { II } \\ \text { Sofort } & 0 & 0 & - & 76.00 \\ \text { Zim. 2 } & & 59.33 & - & 9767\end{array}$

Die IJaten sind Mittelwerte von dreimaligen Versuchen ausgeführt mit je Ioo Samen.

Tabelle 27. Resultate erhalten mit den aus den Früchten mit dem Reifegrad o gesammelten Samen von Solanum (Sorte "Naganasu“), welche teilweise in Talelle 26 benutzt wurden.

$\begin{array}{cccc}\begin{array}{c}\text { Reifegrad der } \\ \text { Frichte zur } \\ \text { Erntezeit }\end{array} & \begin{array}{c}\text { Behandelung } \\ \text { der Früchte }\end{array} & \begin{array}{c}\text { Keimprozente der Samen } \\ \text { zur Samen- } \\ \text { sammelungszeit }\end{array} & \begin{array}{c}\text { ausserhalb der Frucht } \\ \text { 2 Wochen lang gelagert }\end{array} \\ \text { o } & \text { Sofort } & 0 & 0 \\ 0 & \text { Zim. 2 } & 59.33 & 56.00\end{array}$

Die Daten sind Mittelwerte von dreimaligen Versuchen ausgeführt mit je 100 Samẹn.

Tabelle 28. Resultate erhalten mit den aus den Früchten mit dem Reifegrad o gesammelten Samen von Solurirm (Sorte „Naganasu“), welche teilweise im Versuch VII gebraucht wurden.

\begin{tabular}{cccccc}
$\begin{array}{c}\text { Reifegrad der } \\
\text { Frifchte zur } \\
\text { Erntezeit }\end{array}$ & $\begin{array}{c}\text { Behandelung } \\
\text { der Früchte }\end{array}$ & $\begin{array}{c}\text { Keimprozente der Samen } \\
\text { zur Samen- } \\
\text { sammelungszeit }\end{array}$ & $\begin{array}{c}\text { gelagert ausserhalb der Frucht, } \\
\text { I Woche } 2 \text { Wochen }\end{array}$ Wochen \\
o & Sofort & 0 & 0 & 0 & 0 \\
o & Zim. I & 3233 & 40.00 & 23.00 & 31.00 \\
0 & Lich. 2 & 48.00 & 51.00 & 49.00 & 56.00 \\
\hline
\end{tabular}

Die Daten sind Mittelwerte von dreimaligen Versuchen ausgeführt mit je Ioo Samen.

Aus allen erwähnten Versuchsresultaten können wir ableiten, dass die Keimfähigkeit der unreifen Samen in dem Falle zunimmt, wo sie in den Früchten gelagert, sei es zusammen mit der Mutterpflanzen oder von ihr 
getrennt. Obwohl die Zunahme der Keimprozente der ausserhalb der Frucht gelagerten Samen von Capsicum (Sorte "Ônaru") und Cucumis auch in gewissem Grade bemerkbar war, so kam doch das doch geringer vor, als bei dem Falle der innerhalb der gelagerten Früchte befindlichen Samen. Obwohl also diese Zunahme der Keimfähigkeit der Samen teilweise durch die sog. Nachreifung der Samen bedingt sein mag, so muss doch diese Erscheinung aller Wahrscheinlichkeit nach grosstenteils durch den physiologischen Einfluss der Frucht auf die Samen verursacht sein.

Dass die Keimfähigkeit der an den abgeschnitten Mutterpflanzen sitzenden Gramineen-Samen erhöht ist, ist schon von verschiedenen Forschern (Z. B. I, I 5, I9, 22, 23, 30, etc.) nachgewiesen. Doch kann man daraus nicht schliessen, dass die Frucht die Reifung der Samen beeinflusst, weil die untersuchten Gramineen fast kein Fruchtfleisch besitzen.

\section{ZUSAMMENFASSUNG}

1. Die Farben der Capsicum-, Cucumis- und Solanum-Früchte wurden durch Lagerung allmählich tiefer, unabhängig davon, ob die Früchte auf den Mutterpflauzen belassen oder von ihnen getrennt wurden.

2. Im Laufe der Farbenänderung der Früchte wurcle an den darin enthaltenen Samen wahrnehmbar:

a) eine Zunahme des Trockengewichts, und

b) eine Zunahme der Keimfähigkeit.

3. Wenn die Samen in den Früchten, und zwar selbst in den von den Mutterpflanzen getrennten belassen werden, findet also aller Wahrscheinlichkeit nach eine Stoffeinwanderung von der Frucht in die Samen und eine Steigerung der Keimfähigkeit derselben statt.

4. Diese Erscheinung war bei Capsicum deutlicher bei der Fruchtaufbewahrung in besonnter Lage zu erkennen als bei Zimmerlagerung.' Dagegen lagen die Verhältnisse bei Cucumis gerade umgekehrt, während bei Solanum diese Verhältnisse dieselben waren, unabhängig davon ob die Früchte im Zimmer aufbewahrt oder direkt den Sonnenstrahlen ausgesetzt worden waren. Die Frage der Ursache für diesen Unterschied soll hier unerörtert bleiben.

5. Die Keimfähigkeit der Samen, welche nach der Ernte ausserhalb der Frucht gelagert wurden, kann ebenfalls zunehmen. In diesem Falle aber war der Grad im geringeren, als bei den innerhalb der Frucht gelagerten Samen. 


\section{LITERATUR}

I. ANIO, II., Ueber die Produktivität der Reis - und Gerstekörner in verschiedenen Reifegraden (Jap.). Nojishikenjo IIokoku Nr. 17, p. 21, Igor.

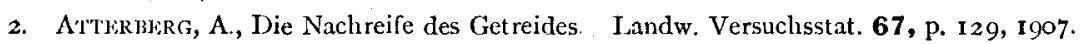

3. Arwool, W. M., $\Lambda$ physiological study of the germination of Avena fatua. Bot. Gaz. 57, p. 386,1914 .

4. CrockFr, W., The mle of seedcoats in delayed germination. Bot. Gaz. 42, p. 265, 1906.

5. -- - Mechanics of dormancy in seeds. Amer. Jour. Bot. 3, p. 98, 1916.

6. I)AVIS, W. E. and E., C. RosE, The effect of external conditions upon the afterripening of the secd of Crataes is Mollis. Bot. Gaz. 54, p. 49, 1912.

7. Eckrrson, S., A physiological and chemical study of afterripening. Bot. Gaz. 55, p. 236, 1913.

8. Fruwirtir, C., Ueber den Sitz des schwersten Kornes in den Fruchtständen bei Getreide " und in den Friichten der IIülsenfrüchte. („WoLlNwy's Forschungen auf dem Gebiete der Agric. Physik“ 15, p. 49, 1892.) Ref. Bot. Centrabl. 52, p. 137, 1892.

9. Golizit, K., Organography of plants. Part II. Oxford 1905.

10. Grafe, V., Frnährungsphysiologisches Praktikum der höheren Pflanzen. Berlin I9I4.

II. IIARRINGTON, G. II. und B. C. IIITE, Afterripening and germination of apple seeds. Jour. Agr. Kes. 23, p. I 53, 1923.

12. I Io'TYR, II, Ueber die Vorgänge bei der Nachreife von Weizen. Landw. Versuchs Stat. 15, p. $356, \mathrm{t} \delta 92$.

13. Ilnwkins, I.. A. and J. R. Magness, Some changes in Florida grapefruit in storage. Jour. Agr. Res. 20, p. 357, 1920.

14. Janson, C., Untersuchungen über die Finlagerung der Reservestoffe in die Hafer und Gerstenkörner beim Reifungsprozess. (Dissert. Jena. 8o, I907.) Ref. Bot. Centrall1. 110, p. 454, 1909 .

15. Kondo, M., Ueber die Nachreife und Keimung verschieden reifer Reiskörner. Ber. des Ohara Inst. für Landw. Fors. 1, p. $3^{6 \mathbf{r}}$, rgi 8.

I6.' Kuliscir, P., Untersuchungen ïber das Nachreifen der Aepfel. Landw. Jahrb. 21, p. 87 I, 1892.

17. Komatsu, S. and M. Ishrmasa, On the biochemical study of the ripening of the Kaki-fruit. Jour. Bioch. Tokyo 3, p. 261, 1924.

18. I.tinRA, J. M., Respiration and breakdown of ripe pears. New Phytolngist 23, p. I31, 1924 .

19. Lucunus, B., (Landw. Versuchs-stat. 4, p. 262, 186z.) Zitiert nach Nobre's Samenkunde, Berlin 1876.

20. Magness, J. R., Investigations in the ripening and storage of Bartlett pears. Jour. Agr. Res. 19, p. 473 , I920.

21. Müit.fr-ThURGaw, Einfluss der Kerne auf die Ausbildung des Fruchtfleisches bei Traubenbeeren und Kernobst. (Jahresber. Versuchsstat. Wadenweil 2,' I893.) Ref. Bot. Centrabl. 54, p. 26, 1893 .

22. NoBBe, F., Samenkunde. Berlin 1876 .

23. Nowackr, A., (Untersuchungen über das Reifen des Getreides. Halle I87o.) Zitiert Nösise's Samenkunde, Berlin I876. 
24. Overhalskr, E. L. and R. H. Taylar, Ripening of pears and apples as modified by extreme temperatures. Bot. Gaz. 69, p. 273 , 1920.

25. Otro, R., Reifestadium bei Aepfeln. (Proskamer, Obst-Bau zeitung. Jahrg. 6, Igor.) Ref. Bot. Centrabl. 88, p. 355, 1901.

26. Pf.effer, W., Physiology of plant. Vol. I. 2 edition. Oxford Igí.

27. Pfenniger, U., Untersuchung der Fridchte von Phaseolus vulgaris, L. Ber. Deut. Bot. Ges. 27, p. 227, 1909.

28. PACK, D. A., Afterripening and germination of Juniperus-seed. Bot. Gaz. 72, p. 32, I92I.

29. Ridgway, R., Calorstandard and Nomenclature. Washington 1912.

30. Siegert, T., (Landw. Versuchsstat. 6, 1869.) Zitiert in NoßBE*s Samenkunde, Berlin 1876 .

31. Wiesner, J., Ueber die Ruheperiode und iber einige Keimungsbedingungen der Samen von Viscum album. Ber. Deut. Bot. Ges. 15, p. 503, I898.

32. Wassilief, N., Eiweisbildung in reifenden Samen. Ber. Deut. Bot. Ges. 26, p. 454, 1908.

33. ZALfSKI, W., Ueber den Umsatz der Phosphorverbindungen in reifenden Samen. Ber. Deut. Bot. Ges. 25, p. 58, 1907.

34. _-_ - Zur Kenntniss der Stoffwechselprozesse in reifenden Samen. (Beih. Bot. Centrabl. 1, I9ı.) Ref. Bot. Centrabl. 117, p. 56, 1911. 